\title{
Liposome: A Novel Drug Delivery System
}

\author{
Ganesh Shankar Sawant ${ }^{1}$, Kiran Vilas Sutar ${ }^{2}$, Akhil S. Kanekar ${ }^{3}$ \\ ${ }^{1,2}$ Final Year B. Pharmacy of Shree Sarasvati Institute of Pharmacy, Tondavali, Kankavali, Sindhudurg, \\ Maharashtra. \\ Dr. Babasaheb Ambedkar Technological University, Lonere, Raigad, Maharashtra. \\ ${ }^{3}$ Assistant Professor in Shree Saraswati Institute of Pharmacy, Tondavali, Kankavali, Sindhudurg, Maharashtra. \\ Dr. Babasaheb Ambedkar Technological University, Lonere, Raigad, Maharashtra.
}

Corresponding Author: Kiran Vilas Sutar

\begin{abstract}
Liposome is a spherical sac phospholipid molecule. It encloses a water droplet especially as form artificially to carry drug into tissue membrane.

It is spherical sac vesicle it consists at least one lipid bilayer. Liposomes are mainly development for drug delivery size and size distribution. The process of sonication (extrusion) is required to obtain small size and narrow size distribution of liposome. The main significant role in formulating of potent drug, improve therapeutic effect. Liposome formulation is mainly design in increasing accumulation at the target site, and then resulting effect is targeted to reduce toxicity. There is various method for liposome formulation depending upon lipid drug interaction liposome disposition mechanismparameters particle size, charge and surface hydration.
\end{abstract}

Liposome is a nanoparticle (size-100nm). Nanoscale drug delivery system using liposome as well as nanoparticle. This technology is for "Rational delivery of chemotherapeutic" drug treatment of cancer. Liposome is use as to study the cell membrane and cell organelles. The advantages of liposome formation using microfluidic approach for bulk-mixing approaches are discussed.

Key Words: - liposome, lipid bilayer, sonication, nanoparticles, particle size, toxicity.

\section{INTRODUCTION}

The name liposome is derived from two Greek words: 'Lipos' meaning far and 'Soma' meaning body. ${ }^{[1]}$ Liposome is a spherical sac phospholipid molecule enclosing a water droplet, especially are formed artificially to carry drug into the tissue. ${ }^{[2]}$

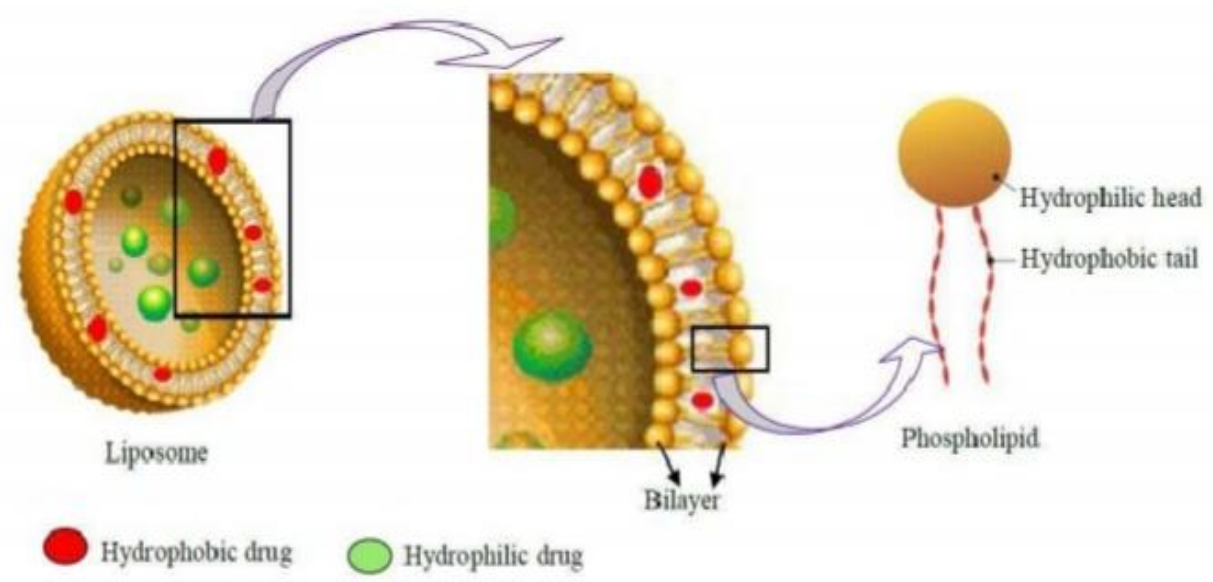

Figure 1. Structure of liposome and lipid bilayer. ${ }^{[9]}$ 
Liposome are also defined as artificial microscopic vesicles consisting of aqueous compartment and surrounded by one or more concentric layer of phospholipid. The sphere like encapsulated a liquid interior contain more substance like peptides, protein, hormones, enzymes, antibiotic, antifungal and anticancer agents. Liposome is small artificial vesicles of spherical shape that can create cholesterol and naturally non-toxic phospholipid. They are depending upon size, hydrophobic and hydrophilic characteristic. Liposome is a spherical vesicles having at least one lipid bilayer.

It is use as vehicle for administration of nutrients as well as pharmaceutical drugs. It shows both characteristics-

1) Hydrophilic head

2) Lipophilic tail ${ }^{[3]}$

\section{- Structural component of liposome- ${ }^{[1-5]}$}

Liposomes are composed lipid bilayer size: - 50-1000nm in diameter that serve as targeted delivery vehicle that contain active biological compound. Liposome most often composed of phospholipid and cholesterol.

- Phospholipid- It is major structural component of liposome. It has the characteristic of excellent biocompatibility and amphiphilic in nature [4]. It contains exist two sorts of phospholipidphosphodiglycerides and sphingolipid. The most common phospholipid is phosphatidylcholine (PC) molecule. Phospholipid is carry both water soluble and lipid soluble drug to target site.

Examples of phospholipid-

1) Phosphatidyl choline (Lecithin) - PC

2) Phosphatidyl ethanolamine (cephalin) $\mathrm{PE}$

3) Phosphatidyl serine (PS)

4) Phosphatidyl inositol (PI)

5) Phosphatidyl glycerol (PG) ${ }^{[1]}$

\section{- Cholesterol- ${ }^{[5]}$}

Cholesterol is one of the other components present in liposome. Cholesterol contain does not bilayer construction but its ability to include into phospholipid membrane (concentration is 1:1 or even 2:1 molar ratio of cholesterol to phosphatidyl choline. The concentration of cholesterol is affecting the particle size of liposome.

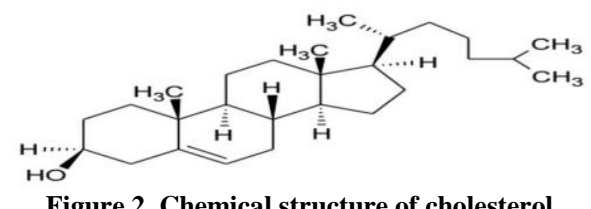

Synthetic lipid 1, 2-palmitoyl-sn-glycero-3phosphatidylcholine (DPPC) and cholesterol

Table 1: - Initial particle size of liposome in corporate with cholesterol at Different ratio. ${ }^{\text {[5] }}$

\begin{tabular}{|ll|l|l|l|l|}
\hline $\begin{array}{l}\text { Component of DPPC } \\
\text { :Cholesterol }\end{array}$ & $\begin{array}{l}\text { DPPC: } \\
\text { Cholesterol 4:0 }\end{array}$ & $\begin{array}{l}\text { DPPC: } \\
\mathbf{4 : 0 . 5}\end{array}$ & Cholesterol & $\begin{array}{l}\text { DPPC: } \\
\mathbf{4 : 1}\end{array}$ & Cholesterol \\
\hline Particle size & & $248.3 \mathrm{~nm}$ & $234.0 \mathrm{~nm}$ & $260.2 \mathrm{~nm}$ & $\mathbf{4 : 2}$ \\
\hline
\end{tabular}

Generally, liposomes are definite shaped spherical sac vesicle with particle sizes ranging from $30 \mathrm{~nm}$ to several micrometers. It shows beside biocompatibility characteristics. This layer is referring to lamellae. ${ }^{[6]}$ Liposome is widely used in cosmetic and pharmaceutical industry. ${ }^{[7]}$ Food farming industries are extensively use of 'liposome encapsulation' to grow delivery system. It can use entrapped unstable compounds (e.g., Antimicrobial, antioxidant, flavors and bioactive element). Liposome can act as a carrier for various drugs it having verisimilar therapeutic action.

\section{- Phase transition temperature of liposome -}

Phase transition temperature $(\mathrm{Tc})$ is temperature at which a membrane changes between the fluid and gelled state. Phase transition of lipid bilayer is most important properties of liposome. The objective of this study is molar ratio of 1,2-dipalmitoyl-sn- 
glycero-3-phosphocholine

(DPPC), hydrogenerated Soy phosphatidyl choline (HSPC) ${ }^{[8]}$, dimyristoyl phosphatidyl choline (DMPC), dioleoyl phosphatidyl choline (DOPC), distearoyl phosphatidyl choline (DSPC), dipalmitoyl phosphatidyethanolamine (DPPE), dipalmitoyl phosphatidyl choline (DPPC) and dipalmitoyl phoshatidyglycerol (DPPG).

Table 2: - phase transition temperature of various phospholipids $^{[9]}$

\begin{tabular}{|l|l|c|}
\hline Name of the phospholipid & $\begin{array}{l}\text { Molecular } \\
\text { weight }\end{array}$ & $\begin{array}{l}\text { Phase-transition } \\
\text { temperature } \\
\left({ }^{\circ} \text { C) }\right.\end{array}$ \\
\hline $\begin{array}{l}\text { Dimyristoyl } \\
\text { phosphatidylcholine (DMPC) }\end{array}$ & 677.94 & 23 \\
\hline Dioleoyl PC (DOPC) & 786.12 & -22 \\
\hline Distearoyl PC (DSPC) & 790.15 & 55 \\
\hline Dipalmitoyl PC (DPPC) & 734.05 & 41 \\
\hline $\begin{array}{l}\text { Dipalmitoyl } \\
\text { phosphatidylethanolamine } \\
\text { (DPPE) }\end{array}$ & 691.97 & 67 \\
\hline $\begin{array}{l}\text { Dipalmitoyl } \\
\text { phosphatidyglycerol (DPPG) }\end{array}$ & 744.96 & 41 \\
\hline
\end{tabular}

Liposome with low Tc (less than $37^{\circ} \mathrm{C}$ ) are fluid like and result leakage of drug content at physiological temperature. But, the high $\mathrm{Tc}$ (greater than $37^{\circ} \mathrm{C}$ ) of liposomes is rigid and less leakage possibility at physiological temperature. Table indicates phase transition temperature of various phospholipids. ${ }^{[9,10,11]}$

\section{History of liposome-}

The liposomes are first described in 1964 by 'A. D. Bangham' and his colleague 'R. W. Thome'. ${ }^{[12]}$ After examining, analyzing and observing the dispersion of phospholipids in water under electron microscope- (Betageri et al., 1993). They found that the phospholipid arranged in automatically and form structure that they referred to as "bag like". A close colleague, Gerald's Weismann" suggests structure called as liposome, which he then defined as "microscopic vesicle composed of one or more lipid bilayer. This are led to large field of research. ${ }^{[13,14]}$

Generally, liposome can be divided into three periods ${ }^{[15]}$

1) Genesis

2) Middle age
3) Modern era

1) Genesis (1968-1975)-

In this period, physio-chemical characterization of liposome is carried out and developed the method of preparation of multilamellar vesicles (MLVs). Liposome are widely use in study the nature of biological membrane.

\section{2) Middle Age (1975-1985)-}

Liposome utility was improving the research and increased the understanding of their stability and interaction characteristics. This period can achieve the discovery of various alternative methods for the preparation of liposome. Also due to availability of vast knowledge about the liposome and their physio-logical properties, their behavior within the body, their interaction with the cells.

\section{3) Modern Era (1985 onwards)-}

Today, liposomes are used successfully in various scientific disciplines, mathematical and theoretical physics, biophysics (properties of cell membrane and their channels), chemistry (photosynthesis, catalysis and energy conversion), colloid science (stability, thermodynamics), biochemistry (photosynthesis, function of membrane protein), biology (excretion, cell function, gene delivery and function).

\section{Advantages of liposome- ${ }^{[2,16]}$}

1) Can carry both water and lipid soluble drugs.

2) Non-ionic in nature.

3) Liposome is biocompatible, completely biodegradable, non-toxic, and nonImmunogenic.

4) Suitable for delivery of hydrophobic, amphipathic and hydrophilic drug.

5) Protect encapsulated drug from the external environment.

6) Liposome reduces toxicity and increase stability via encapsulation.

7) They are increase activity of chemotherapeutic drug. 
8) Biodegradable drug can be stabilized from oxidation.

9) Reduce exposure of sensitive tissues to toxic drugs.

10) Improve protein stabilization.

11) Control hydration.

12) Provide sustained release.

13) Targeted drug delivery or site specific drug delivery.

14) Can be administered through various routes.

\section{Disadvantages of liposome- ${ }^{[2,17]}$}

1) Production cost is high.

2) Leakage and fusion of encapsulated.

3) Short half-life.

4) Stability problems.

5) Allergic reaction may occur to liposome constituents.

6) Problem to targeting to various tissues due to their large size.

7) Phospholipid undergoes oxidation, hydrolysis.

\section{Classification of liposome- ${ }^{[3,18]}$}

1) Classification of liposome depending upon size and shape

a) Multilamellar vesicles (MLV)

b) Large unilamellar vesicles (LUV)

c) Small unilamellar vesicles (SUV)

2) Classification of liposome according to composition

a) Conventional liposome

b) $\mathrm{PH}$ - sensitive liposome

c) Cationic liposome

d) Long circulating liposome

e) Immuno- liposome

3) Classification of liposome depending upon production method

a) Passive loading technique

b) Mechanical dispersion method

i) Lipid hydration by hand shaking or freeze drying

ii) Micro emulsification

iii) Sonication

iv) French pressure cell

c) Solvent dispersion method i) Ethanol injection

ii) Ether injection

iii) Double emulsion vesicle

iv) Reverse phase evaporation

d) Detergent removal method

i) Dialysis

ii) Detergent removal of mixed micellar

iii) Dilution

e) Active loading technique

1) Depending upon size and shape- ${ }^{[3,9,19}$

a) Multilamellar vesicle (MLV)-

Multilamellar vesicle are generally size between '100- $1000 \mathrm{~nm}$ ' and it consist of two or more than two bilayers. The method of preparation of multilamellar vesicle is very simple, which include in thin- film hydration method/ hydration of lipids in excess of organic solvent. They are very long storable because they are mechanically stable. It is rapidly cleared by "Reticulo Endothelium System" (RES) cell.

\section{b) Large unilamellar vesicle (LUV)-}

The large unilamellar vesicles of liposome consist of a single bilayer or single lamella. LUV size is ' $>0.1$ micrometer and can reach size up to 1000 $\mathrm{nm})$. They have mainly high efficiency of encapsulation, since ability to hold large volume of solutions in their cavity. They are similar to multilamellar vesicle. Large unilamellar vesicles are prepared from various methods like ether injection, reverse phase evaporation technique and detergent dialysis.

\section{c) Small unilamellar vesicle (SUV)-}

Small unilamellar vesicles are generally smaller size $(<0.1$ micrometer $)$ as compared to multilamellar vesicle and large unilamellar vesicle. Small unilamellar vesicles contain single bilayer. SUV are prepared from solvent injection method (ethanol and ether injection). 

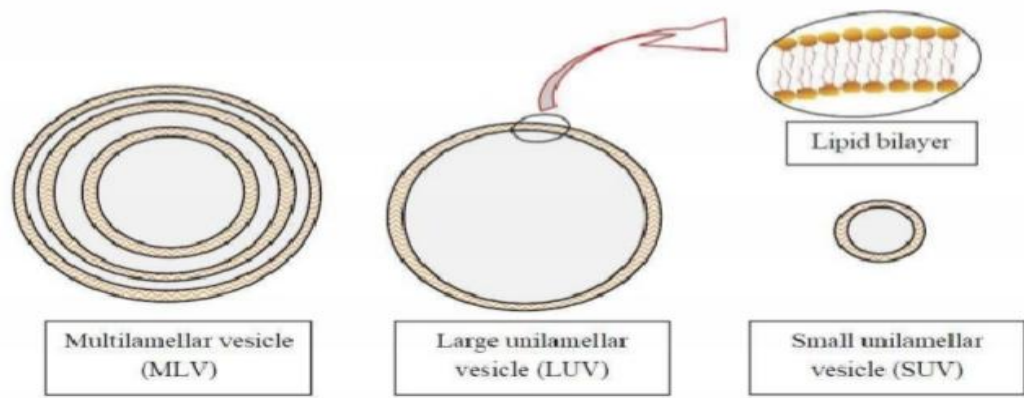

Figure 3. Classification of liposome based on size and number of bilayer. ${ }^{[9]}$

2) Classification of liposome depending upon composition-

The liposome membrane is normally constituted from natural components found in membrane of living cells, but these constituents include in synthetic materials.

\section{- Conventional liposome-}

Conventional liposome is composed of natural phospholipid or lipid such as sphingomyelin, egg phosphatidylcholine, 1-2disteroryl-snglycero-3-phosphatidyl choline (DSPC) and monosialonganglioside. Liposome contain positive and negative charge have been reported to have shorter half- lives, toxic and rapidly remove from systemic circulation. ${ }^{[21,22,23]}$ These liposomes are mainly use for targeting of the 'Reticuloendothelial system'(RES). Conventional liposome is use mostly use as compare to other types because shortens the circulation times of the liposome. ${ }^{[3]}$ To increase circulation time, liposome surface coated with a hydrophilic polymer, repulsive forces of liposome and serum-components. ${ }^{[24]}$

Conventional liposome- based technology is first generation of liposome to be used in pharmaceutical applications. ${ }^{[25,}$ 26, 27] Several attempts to overcome their challenges have been made, specifically manipulation of the lipid membrane.

\section{- pH- sensitive liposome-}

$\mathrm{PH}-$ sensitive liposome is composed of oleic acid (OA), phosphatidyl ethanolamine (PE), cholesterol hemisuccinate (CHEMS). ${ }^{[3]}$ It has been focus is development of strategies to increase ability of liposome to mediate intracellular delivery of biological active molecules. This result modified form of liposome is called $\mathrm{pH}$ - sensitive liposome. $\mathrm{pH}$ - sensitive liposomes are stable at physiological $\mathrm{pH}$ (pH- 7.4) but such condition leading to release of their aqueous contents- undergo destabilization and fusogenic property under acidic conditions. Different classes of $\mathrm{pH}$ - sensitive liposome based on mechanism of triggering $\mathrm{pH}$ sensitivity (Torchilin et al. 1993; Drummond et al 2000). The most commonly established hypothesis involves the blend of phosphatidylethanolamine (PE) and its derivative (containing acidic group, e.g. carboxylic group) that act as a stabilizer at neutral $\mathrm{pH}$. ${ }^{[28]}$

$\mathrm{pH}$ - sensitive liposomes are lipid composition that can be destabilized when the external $\mathrm{pH}$ is change of usually from neutral or slight alkaline $\mathrm{pH}$ to an acidic $\mathrm{pH}$. In cell culture $\mathrm{pH}$ sensitive liposome can increase the delivery of proteins, fluorescent markers, cytotoxic substance, RNA and DNA into the cytoplasm. ${ }^{[29]}$

\section{- Cationic liposome-}

Cationic liposomes are composed dimethyl-dioctaatidecyl ammonium bromide (DDAB), dioctadecyldimethyl ammonium chloride (DOGS), 2,3-dioleoyloxy-N(2(spermine carboxamido)-ethyl)-N, Ndimethyl-1-propanaminiu fluoracetate (DOSPA) 1,2 dioleoyloxy-3(trimethylammonio)propane(DOTAP),1,2di mrystyloxypropyl-3-dimethyl-hydroxethyl ammonium bromide (DORIE) combined with dioleoylphosphatidyl ethanolamine (DOPE). These liposomes are highly toxic can cause short lifespan, thus limited then to 
local administration. They are mostly use for delivery of macromolecules (negatively charge ${ }^{[30]}$ and delivery of DNA and RNA (New, 1990). ${ }^{[3]}$ Fusion between cationic vesicles and cell surfaces can deliver the DNA across the plasma membrane. This process bypasses the route of endosomallysoma route which leads to degradation of formulation of an anionic liposome. ${ }^{[31]}$

\section{- Long circulating liposome-}

Long circulating liposome can prepare by coating liposome surface with a hydrophilic layer of oligosaccharides, glycoproteins, synthetic polymers in order to make liposomes. Scavenger cells of the mononuclear phagocyte system. ${ }^{[32]}$

Long circulating liposome are widely use in biomedical in-vitro and invivo studies and clinical practice. The liposome is very useful tools, especially for tumor targeting therapy. Long circulating liposome exhibit dose-independent, nonsaturable, long-linear kinetics and Increased bioavailability. ${ }^{[28]}$

\section{- Immuno-liposome (ILs)- ${ }^{[33]}$}

Immuno-liposome (ILs) is generated by coupling antibodies either directly to liposome lipid bilayer in the presence of PEG chains (type 1 liposome) or to the distal end of PEG chains (type 11 liposome). Coupling antibodies to the lipid bilayer of PEGylated. Liposome can result reduce antigen binding depending on amount of PEG and length of the PEG chains. ${ }^{[34,35]}$ ILs antigen binding can be restored by coupling antibody to the terminus of PEG chain. ${ }^{[36]}$ In conclusion, whole antibodies are several disadvantages of for the generation of ILs.

\section{3) Classification of liposome depending} upon production method-

\section{A) Passive loading technique-}

Passive loading in which liposome are formed concurrently with drug loading. In that hydrophilic compounds are distributed homogeneous in the aqueous phase (both inside and outside the liposomes), hydrophobic drugs are retaining inside the lipid bilayer of liposome, when working with water soluble drugs. The drug is firstly dissolved with lipid in organic solvent, followed by solvent evaporation method to prepare drug containing thin film. After prepare thin film hydrated with on aqueous phase to prepare liposome. When the loading of water soluble drugs, the film of lipid is dispersed in a drug-containing aqueous phase. ${ }^{[37]}$

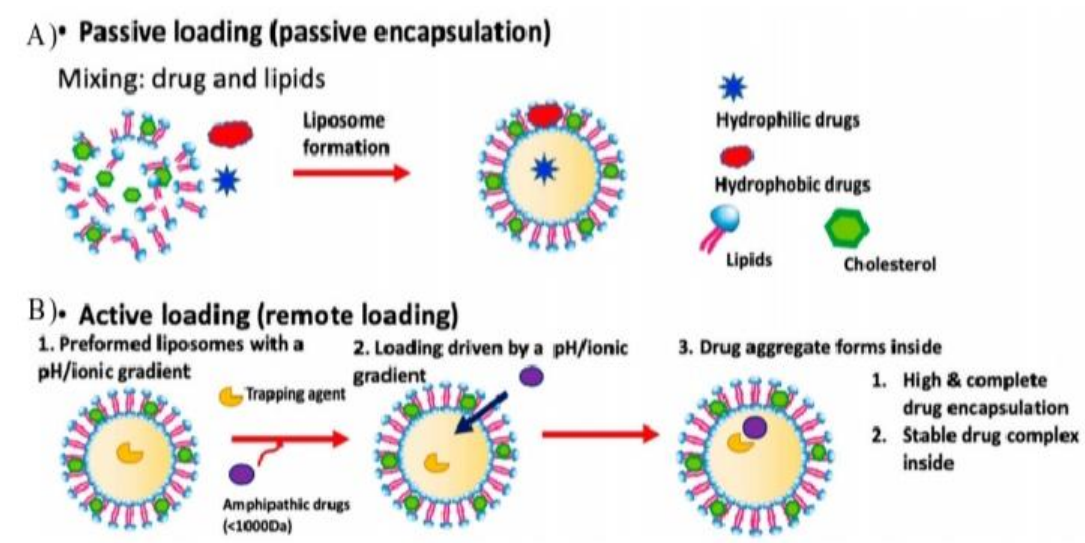

Figure 4. Passive loading and active loading involves liposome formation. ${ }^{[37]}$

\section{a) Mechanical dispersion method-}

Aqueous volume (5-10\%) enclosed using this method, which is small proportion of total volume used for swelling. Therefore, large quantity of water- soluble compound is wasted during swelling, On the other hand lipid soluble compound can be encapsulated to $100 \%$ efficacy. It provides they are not present in quantities that are 
greater than the structural component of the membrane. ${ }^{[38]}$

\section{- Lipid hydration by hand shaking- Step (1)-}

To prepared firstly lipid mixture of different phospholipid and charge components in chloroform: methanol (2:1 $\mathrm{v} / \mathrm{v})$ solvent mixture. Then introduce into a round bottom flask a ground glass neck. This flask is attached to rotary evaporator (rotated at $60 \mathrm{rpm}$ ). The organic solvent is evaporated at about $30^{\circ} \mathrm{C}$ or about transition temperature of lipid. The evaporator is isolated from the vacuum source by close the tip. The nitrogen is introduced into the evaporator and the pressure of cylinder is gradually raised up to no difference between inside and outside the flask. Remove the flask from the evaporator and fixed on lyophilizer to remove residual solvents. ${ }^{[38,39]}$

\section{Step (2) - Hydration of lipid layer}

After removal from lyophilizer, the flask flushed with nitrogen; $5 \mathrm{ml}$ saline phosphate buffer is added. The flask is again attached to evaporator and flushed with dinitrogen (N2). The evaporator are rotated at room temperature and pressure at same speed (for below $60 \mathrm{rpm}$ ). The flask is stop rotate after 30 minute or until all lipid has been removed from the wall of the flask and has given homogeneous milky suspension. The suspension is allowed to stand for 2 hours at room temperature or at a temperature above transition temperature of the lipid in order to complete the swelling process to give MLVs (Multilamellar vesicle). ${ }^{[38]}$

\section{- Sonication -}

Sonication is a process in which sound waves are used to agitate particle in solution. Such disruption can be used to mix solutions, speed the dissolution of a solid into a liquid and remove dissolved gas from liquid. ${ }^{[40]}$

Sonication is method in which MLVs are transformed to small unit lamellar vesicles (SUVs). The ultrasonic irradiation is provided to convert MLVs to SUVs. There are two method used,

- Probe sonication method

- Bath sonication method ${ }^{[38]}$

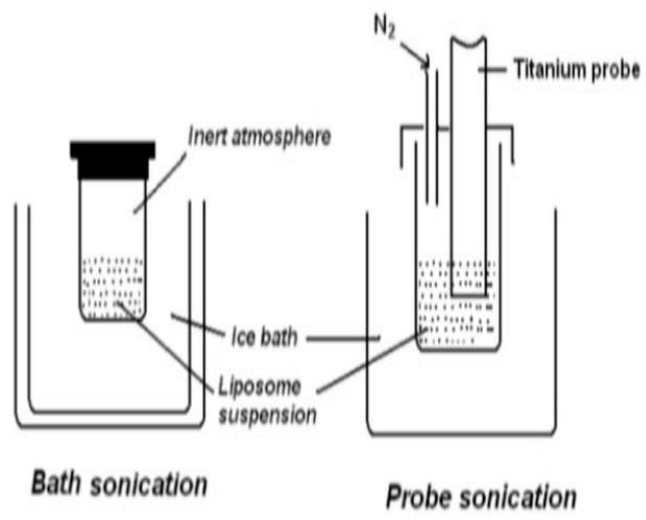

Figure 5. Sonication apparatus. ${ }^{[16]}$

\section{- Probe sonication method-}

The probe sonicator is used for high energy in small volume (e.g. high concentration of lipid or viscous aqueous phase). ${ }^{[41]}$ The tip of sonicator is directly immersed into the liposome dispersion is very high in this method. The dissipation of energy at the tip results in local overheating. Then vessel must be immersed into an ice bath. Throughout, the sonication up to 1 hour more than $5 \%$ of the lipids can be deesterify. Also, with the probe sonicator, titanium will slough off and contaminate the solution. $^{[19]}$

The disadvantages of probe Sonicator is contamination of preparation with metal from tip of probe by this method SUVs are formed. They are purified by ultra-centrifugation. ${ }^{[16]}$ Bath sonication is most common instrumentation for preparation of SUV. ${ }^{[41]}$ Probe- sonication is commonly used to homogenize liposome formulations; it is necessary to investigate its influence on drug entrapment efficiency (EE) of liposome. ${ }^{[42]}$

\section{- Bath sonication method-}

The bath sonicator is used for large volume of dilute lipids. ${ }^{[40]}$ The dispersion of liposome in a tube is placed into a bath 
sonicator. Controlling the temperature of the lipid dispersion. This method is easier to sonication the dispersion directly using tip. Material being sonicated and place into sterile container, under an inert atmosphere. Then lipid bilayer of the liposomes can fuse with other bilayers, thus delivering the liposome contents. By making liposomes in a solution of DNA or drug they can be deliver lipid bilayer. ${ }^{[19]}$

\section{- French pressure method-}

This method is based on mechanism of high pressure. This method used to preparation of 1-40 $\mathrm{ml}$ of homogeneous unilamellar liposomes of intermediate size $(30-80 \mathrm{~nm}) .{ }^{[43]}$ This liposome is more stable compared to the sonicated liposomes. This method is some drawbacks are that initial high cost for the pressure cell. Liposome prepared by this method having less structural defects compared to sonicated liposome. ${ }^{[38]}$

\section{b) Solvent dispersion method-}

In these method can be dissolving the lipid and other constituents of the liposome membrane in other solution. The aqueous phase is added to resulting solution. In this aqueous phase contain material which is to be entrapped. ${ }^{[16]}$ Solvent dispersion method involving ether injection method, ethanol injection method, and reverse phase evaporation method. ${ }^{[44]}$

\section{- Ether injection method- ${ }^{[45,46,47]}$}

Ether injection, solution of lipid is dissolve into ether or diethyl ether or methanol mixture. These mixtures slowly injected into aqueous solution of the material to be encapsulation at $55-65^{\circ} \mathrm{C}$ or under reduce pressure. Then ether is removed with the help of vacuum leads to formation of liposome.

\section{- Ethanol injection method- ${ }^{[43]}$}

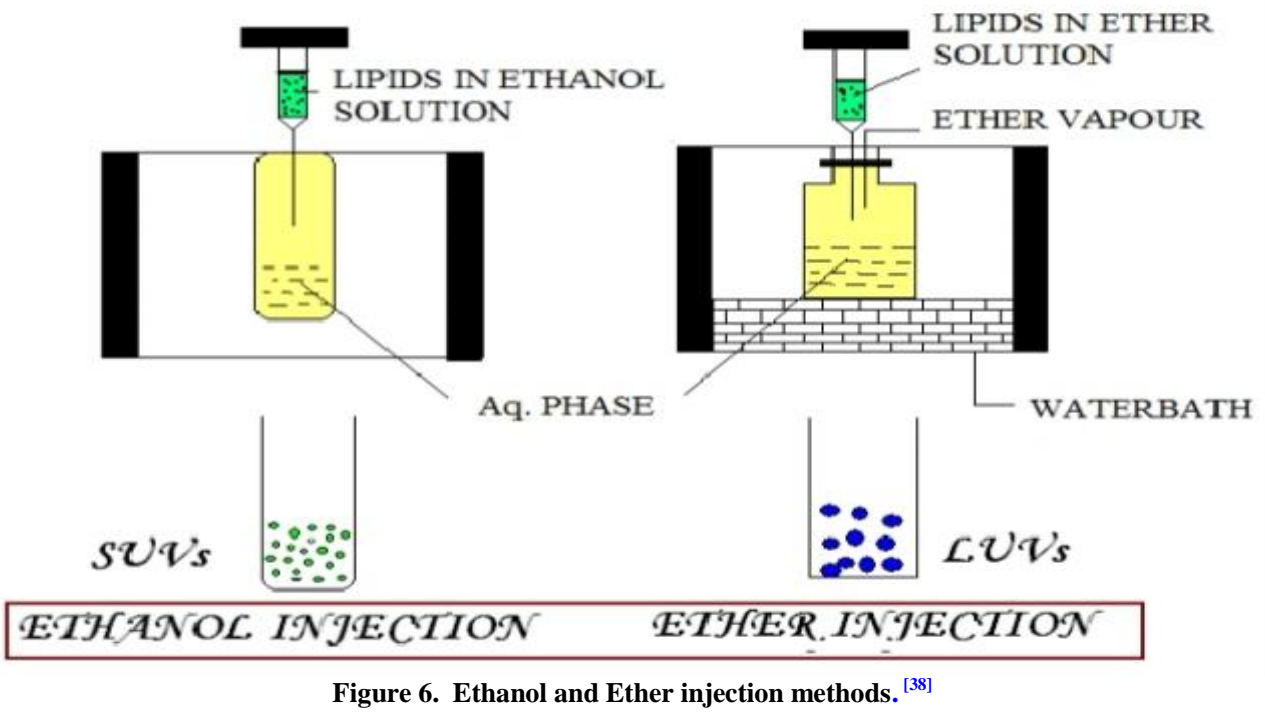

This is simple method. In this method an ethanol solution of the lipid is directly injected rapidly to an excess of saline through a fine needle. The solution of ethanol is diluted in water and phospholipid molecules. They are dispersed evenly through the medium. This procedure yields a high proportion of SUVs (about $25 \mathrm{~nm}$ diameter).

- Reverse phase evaporation method- ${ }^{[45,}$ 48]

The water in oil emulsion is formed by sonication of two phase system. It contains phospholipid in organic solvent 
(diethyl ether) and aqueous buffer. This mixture of lipid is added to round bottom flask. The organic solvent is removing under pressure by a rotary evaporation. The system is purge with nitrogen and lipids are re-dissolved in the organic phase. Diethyl ether and isopropyl ether are the solvent of choice after the lipids are re-dissolved the emulsion are obtaining and then the solvents are evaporated by evaporation of semi solid gel under reduce pressure ${ }^{[45]}$, at $20-25^{\circ} \mathrm{C}$ rotating at approximately $200 \mathrm{rpm}$. A viscous gel forms and an aqueous suspension appears. Add excess water or buffer and evaporate the suspension for an addition 15 minute at $20^{\circ} \mathrm{C}$ to remove traces of solvent. Dialyze the preparation, and pass through 4B column or centrifuge. [48] Resulting liposome are called 'reverse phase evaporation vesicle' (REV).

\section{c) Detergent removal method- ${ }^{[2,49]}$ - Dialysis-}

The detergent at their critical Michelle concentration (CMC) is used to solubilize lipids. The detergent is detached, the micelles in phospholipid and last combine to form LUVs. The detergent can be removed by dialysis. ${ }^{[2,50,51]}$ The main benefit of detergent dialysis method is formation of liposome populations which are homogeneous in size. The main disadvantages of this method are possibility of retention of traces of detergents into the liposome. ${ }^{[49]}$

- Detergent (cholate, alkyl glycoside, Triton X-100) removal of mixed micelles (absorption)-

Detergent absorption is attained by shaking of mixed micelle solution with beaded organic polystyrene absorbers such as XAD-2 beads (SERVA Electrophoresis GmbH, Heidelberg, Germany) and Biobeads SM2 (Bio-Rad Laboratories, Inc., Hercules, USA). The benefit of detergent absorber is removal of detergent at very low CMC. ${ }^{[2]}$

\section{- Dilution-}

The dilution of aqueous mixed micellar solution of detergent and phospholipids with buffer. The size of micellar and polydispersity is fundamentally increase. ${ }^{[2]}$

\section{B) Active loading technique- ${ }^{\text {[37] }}$}

In active loading, liposomes are first generated containing a transmembrane gradient, i.e. aqueous phase inside and outside the liposomes are different. Subsequently, an amphipathic drug is dissolved in exterior aqueous phase can permeate the phospholipid bilayer. After permeation interaction with trapping agent and core to lock- in the drug.

In 1976, Deamer and Nicols ${ }^{[52,53]}$ demonstrate that a $\mathrm{pH}$ gradient could be utilized to load catecholamine into liposomes, Resulting stable drug retention in vitro. ${ }^{[37]}$

\section{- Evaluation of liposome- ${ }^{[16,54-58]}$}

Liposomal processing and formulation for specified purpose are characterized to ensure their predictable in vivo and in vitro performance. The characterization parameters for purpose of evaluation could be classified into three categories.

1) Physical characterization.

2) Chemical characterization.

3) Biological characterization.

\section{1) Physical characterization: -}

Physical characterization evaluates various parameters include size, shape, surface features, release profile and phase behaviors.

\section{2) Chemical characterization: -}

It includes study of purity and potency of various lipophilic constituents.

\section{3) Biological characterization: -}

They are useful in safety and suitability of formulation for therapeutic application. ${ }^{[16]}$ 
Table No.3 Liposome characterization ${ }^{[54]}$ Physical characterization

\begin{tabular}{|c|c|}
\hline Characterization parameters & Instrument for analysis \\
\hline Vesicle shape and surface morphology & TEM and SEM \\
\hline Vesicle size and size distribution & Dynamic light scattering TEM \\
\hline Electrical surface potential and surface pH & Zeta potential measurement and pH sensitive probes. \\
\hline Surface charge & Free flow electrophoresis \\
\hline Lamellarity & P31 NMR \\
\hline Phase behavior & DSC, Freeze fraction electron microscopy \\
\hline Percent capture & Mini column centrifugation \\
\hline Drug release & Diffusion cell/ dialysis \\
\hline \begin{tabular}{|c|c|}
\hline \multicolumn{2}{|c|}{ Chemical characterization } \\
\hline Characterization parameters & Instrument for analysis \\
\hline Phospholipids concentration & HPLC/ Barret assay \\
\hline Cholesterol concentration & HPLC/ Cholesterol oxide assay \\
\hline Phospholipids per oxidation & U.V Observation \\
\hline pH & pH meter \\
\hline Osmolarity & Osmometer \\
\hline Piological characterization \\
\hline Pyrogenicity & Instrument for analysis \\
\hline Animal toxicity & Aerobic/ Anaerobic culture \\
\hline Characterization parameters & Rabbit fever response \\
\hline Sterility & Monitoring survival rats. \\
\hline
\end{tabular}
\end{tabular}

Some parameters are ${ }^{[16,45]}$

Vesicle shape and lamellarity-

Vesicle shape can be determined using Electron Microscope Technique. Lamellarity of vesicles (number of lipid bilayer present in liposome) is determined by using Freeze-Fracture Electron Microscopy and P31 Nuclear Magnetic Resonance Analysis.

\section{B) Vesicle size and size distribution-}

Determination of vesicle size and size distribution are various technique are available. i.e. Light microscopy, Fluorescent microscopy, Electron microscopy (specially Transmission Electron microscopy), Field Flow Fractionation, Gel permeation, Laser light Scattering Photon Correlation Spectroscopy and Gel exclusion. In these microscopy, Electron Microscopy are mostly use to determination of size range of liposome.

\section{a) Microscopic Technique-}

\section{- Optical Microscopy-}

The microscopic method includes use of Bright-field, Phase-Contrast microscope and fluorescent microscope. It is useful in evaluating size range of large vesicles. ${ }^{[59,60]}$

\section{- Negative stain TEM-}

Electron microscopic technique is used to determine liposome size range and shape are mainly negative stain TEM and scanning electron microscopy. This technique is less preferred. Negative stain electron microscopy visualizes bright areas against dark background. The negative stain used in TEM (Transmission electron microscopy) analysis are ammonium molybdate or phosphotungstic acid (PTA) or uranyl acetate. The nature of uranyl acetate is cationic and both ammonium molybdate, and phosphotungstic acid (PTA) is anionic. ${ }^{[16,45]}$

\section{- CRYO-Transmission Electron Microscopy Techniques (CRYO-TEM)- [59]}

This technique is used to study of surface morphology and size of vesicles.

\section{b) Diffraction and Scattering Technique- \\ - Laser light scattering photon correlation spectroscopy-}

Photon correlation spectroscopy (PCS) is analysis of time dependence of fluctuation intensity in scattered laser light due to 'Brownian motion' of particles in solution or suspension. Since small particles diffuse more rapidly than large particles, the rate of fluctuation scattered light intensity varies accordingly. The translation diffusion 
coefficient (D) can be measured and used to determine the mean hydrodynamic radius (RHSS) of particles using Stoke Einstein Equation. Using this technique measured the particles in range of about $3 \mathrm{~nm}$.

\section{c) Hydrodynamic Technique-}

This technique includes Gel permeation and Ultracentrifuge. Exclusion chromatography on large pure gels was introduced to SUVs from MLVs. The size of large vesicle is 1-3 micrometer diameter usually fail to enter the gel and are retained on top of column. A thin layer chromatography system is used to determination of size distribution of liposome preparation. However, it was not reported if this procedure was sensitive to a physical blockage of pores of the agars gel as is the more conventional column chromatography. ${ }^{[16,45]}$

\section{d) Zeta potential determination-}

The zeta potential was evaluated by the determination of electro mobility of the 90c angle. The measurement was performed in triplicate using the 3000 HS Zeta-Seizer equipment. The sample is diluted with suitable diluents for the potential determination. ${ }^{[45,61]}$

\section{- Recent approaches-}

\section{1) Interferon- gamma - ${ }^{[62]}$}

Liposome as sustain release system for human interferon-gamma. Interferongamma (IFNgamma) has proven to be a promising adjuvant in vaccines against cancer and infectious disease. However, due to its rapid bio degradation and clearance, its efficacy is severally reduced. The liposomal association IFNgamma produce prolong residence time. Human IFNgamma can be formulate in large and multilamellar liposomes with high association efficiency $(>80 \%)$ and preservation of bio bioactivity. A critical parameter is the inclusion of negatively charged phospholipids to obtain a high liposomal efficiency, which is dominated by electrostatic interactions.
In the present study, various liposomal formulations of recombinant human IFN gamma (hIFN gamma), differing in lipid composition, prepared via the film hydration method. The characterized in vitro regarding association efficiency and bioactivity, and in vivo regarding cytokine release kinetics after subcutaneous (S.C) administration into mice. IFN gamma, reducing the initial burst release. Increasing the rigidity of the liposome bilayer also resulted in reduction of the burst release and increase in the residence time of the protein at the S.C. administration site as compared to the free cytokine. The liposomal IFN gamma may strongly depend on the release of cytokines in vivo, may contribute to a rational design of liposomal- cytokine adjuvants in vaccines against cancer and infectious diseases. ${ }^{[62]}$

\section{2) Stealth liposome- ${ }^{[63]}$}

Stealth liposome technology is one of the most used liposome-based system for delivery of active molecules. [64,65] This liposome was developed to overcome most of the challenges encountered by conventional liposome technology. Such as, inability to evade interception by the immune system, toxicity due to charged liposome, low blood circulation half-life, and steric stability. ${ }^{[65,66,67]}$ The strategy of stealth liposome was achieved by modifying the surface of the liposome membrane, a process that was achieved by engineering hydrophilic polymer conjugate. ${ }^{[68]}$ The hydrophilic polymer such chitosan, silkfibroin, polyethylene glycol (PEG) and polyvinyl alcohol (PVA). ${ }^{[69,70]}$ The advantages to polymeric conjugate were considered such as high biocompatibility, low immunogenicity, nanotoxicity, and antigenicity. ${ }^{[64,69]}$

e.g. PEGylated liposomal doxorubicin (DOXIL/Caelyx).

\section{3) Temporary depot Polymeric-Based systems for liposomal coupling ${ }^{[63]}$ \\ Polymer-based system, such as hydrogel or prefabricated scaffolds have}


been used as depots for drugs, regenerative cells, proteins, growth factor, and preencapsulated drug-loaded liposome for sustained release. ${ }^{[71,72,73,74]}$ Various polymer have been researched for this application based on their properties such as biodegradability, biocompatibility, nanotoxicity, and synthetic biodegradable polymeric systems such chitosan, Carbopol, and polyvinyl alcohol. This is temporary depot-forming agents. ${ }^{[75,76]}$

\section{1) Injectable polymeric scaffolds}

The strategy for generating an ideal depot for an active compound or bioactive molecule-loaded liposome. The benefit of in local drug retention and sustained release over prolong time has recently received much attention in both pharmaceutical and bioengineering research. ${ }^{[74,77]}$ The in-situ forming injectable polymer are most successful, since it was able to encapsulate protein or bioactive molecules or pre-encapsulated drug-loaded liposomal formulation (liquid form). ${ }^{[78,79]}$ This solution or suspension mixture injected into the target organ with a needle to form a semisolid scaffold and finally an implant. The successfully shifting from liquid formulation to semisolid and finally an implant was a result various desirable polymeric properties and stimulating agents such as water, light, temperature and $\mathrm{pH} .{ }^{[80]}$ Since the hydrogel were composed of natural or synthetic biodegradable polymers, bioactive molecules were released via passive diffusion, and polymeric degradation. ${ }^{[81]}$

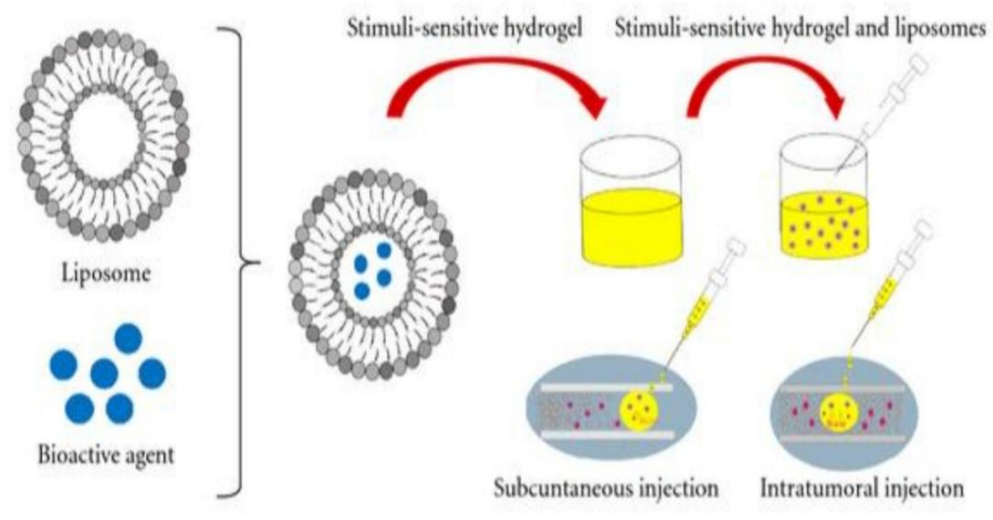

Figure 7. Pre-encapsulated drug-loaded liposomes incorporated within an injectable hydrogel-based system. ${ }^{[63]}$

4) Fusogenic liposomes and antibodymediated liposome in cancer therapy- ${ }^{[2]}$

Fusogenic liposome are used in cancer and tumor treatment. Liposome has been infrequently well-known that a powerful anticancer drug, especially one that targets the cytoplasm or cell nucleus. It does not work due to the low permeability across a plasma membrane, degradation by lysosomal enzymes through an endocytosisdependent pathway. Fusogenic liposome is use in novel drug delivery system, ultimate leading to the induction of ability of anticancer drug. Fusogenic liposome are poised of the Ultraviolet inactivated Sendai virus and conventional liposomes. Fusogenic liposome effectively and directly deliver their encapsulated contents into the cytoplasm by using fusion mechanism of the Sendai virus. The fusogenic liposome is a good vehicle to deliver of drug in cytoplasmic membrane. Liposomal drug delivery system provides consistent formulation, better pharmacokinetics action and make a degree of 'passive' or 'physiological' targeting to tumor tissue.

\section{5) Exparel (liposomal bupivacaine)-}

The majority most patients experience significant pain produce opioid experience have ministry of postsurgical pain. Exparel helps to multimodal therapy was decrees concomitant opioid events. ${ }^{[83]}$ Exparel bupivacaine treatment in 
symptomatic irreversible pulpitis, endodontic, debridement is a predictable method to pain relieve an unexplored treatment in the in the case of long acting anesthetic to reduce pain. ${ }^{[84]}$ A liposomal bupivacaine for postoperative pain management five under want procedure including reconstruction, augmentation, mammoplasty, abdominal wall, reconstruction was pain managed with liposomal bupivacaine compared with traditional pain management. exparel liposomal bupivacaine are prolong pain relief and reduce postsurgical as well as postoperative pain. ${ }^{[85]}$

\section{- Application}

1) Cancer chemotherapy.

2) Gene therapy.

3) Liposomes as carriers for vaccines.

4) Liposomes as carrier of drug in oral treatment.

5) Liposomes for pulmonary delivery.

6) Sustained release drug delivery.

7) Intracellular drug delivery.

8) Lysosomal storage disease.

9) Ophthalmic delivery of drugs.

10) Cell biological application.

11) Mental storage disease. ${ }^{[16,17,60]}$

\section{CONCLUSION}

The liposomal drug delivery is very effective in the delivery of genes and vaccines due to their adjuvant property and tumor targeting ability. Liposomal drug delivery is best tool for targeting of brain. Liposome are made up of lipid bilayer. The ability of liposome to carry both hydrophilic as well as hydrophobic drug for site specific delivery and produce long term effective therapy. Liposome are easily cross 'Blood brain barrier' (BBB) as compared to other dosage form because $\mathrm{BBB}$ is made up of lipid barrier.

\section{ACKNOWLEDGEMENT}

We would like to thank advisor assistant professor Mr. Akhil Kanekar sir for their able guidance, motivation, and support. Also thanks for giving us an opportunity to express ourselves via these review article.

Conflict of Interest: None

\section{Source of Funding: None}

\section{REFERENCES}

1. Hadis D., Ali E., Mohammad K., et al. Application of Liposome in Medicine and Drug delivery. An International Journal of Artificial Cells, Nanomedicine, and Biotechnology. 2016; 44(1): 381-391.

2. Abolfazl A., Soodabeh D., Younes H., et al. Liposome: Classification, Preparation, and Application. Nanoscale Research Letters. 2013; 8: 102.

3. Rajiv Kumar A., Vivek Kumar, Rishi pal, et al. Liposome: A novel approach as a carrier. World Journal of Pharmaceutical Research. 2018; 7(3): 323-342.

4. Jing L., Xulin W., Ting Z., et al. A Review on phospholipids and their main application in drug delivery system. Asian Journal of Pharmaceutical Science. September 2015; 10: 81-98.

5. Li-Ping T., Hong-Jen L., Yi-You H., et al. Liposome incorporated with cholesterol for drug release triggered by Magnetic field. Journal of Medical and Biological Engineering. 2007; 27(1): 29-34.

6. Chrai S.S., Murai R., Imran A. Liposome: A Review. Biopharma. 2001; 14(11): 10-14.

7. Devender M. Sharma, Aashish A., E Ali, et al. An updated review on: Liposome as drug delivery system. Pharmatutor. 2018; 6(2): 50-62.

8. J. Chen, D. Cheng, Jun Li, et al. Influence of lipid composition on the phase transition temperature of liposomes composed of both DPPC and HSPC. Drug Dev Ind Pharm. 2013; 39: 197-204.

9. Kalepu S., K.T. Sunilkumar, Sudheer Betha, et al. Liposomal drug delivery system- A Comprehensive Review. Dept of Pharmaceutical Technology. 2013; 5(4): 6275.

10. Sharma, A., Sharma, U.S. Liposomes in drug delivery: Progress and limitations. Int J Pharm. 1997; 154: 123-140.

11. Vyas S. P., Khar R. K. Targeted and controlled drug delivery: Novel carrier system. CBS Publishers. 2002; p.173-248. 
12. A. D. Bangham. Liposome: The Babraham Connection. Chemistry and Physics of Lipids. September 1993; 64(1-3): 275-285.

13. Kakoli S., Shubhadeep B., Mahitosh M. Liposome as a drug delivery system. In book: Bilogical and Pharmaceutical Applications of Nanomaterials. CRC press publishers. 2019; p.53-100.

14. Bangham A. D., Standish M. M., Weissmann G. The action of steroids and streptolysins on the permeability of phospholipid structures to cations. Journal of Molecular Biology. 1965; 13(1): 253259.

15. Sharma Vijay K., Mishra D. N., Sharma A. K., et al. Liposome present prospective and future challenges. International Journal of Current Pharmaceutical Review and Research. 2010; 1(2): 6-16.

16. Shashi K., Satinder K., Bharat P., et al. A Complete Review on Liposome. International Research Journal of Pharmacy. 2012; 3(7): 10-16.

17. Ramkrishna S., Sunit K. S., Soumyaranjan S. Liposome as drug delivery system: A brief review. International Journal of Research in Pharmaceutical Science. 2014; 5(4): 309-321.

18. Mansoori M. A., Agraval S., Jawade S., et al. A Review on Liposome. International Journal of Advance Research in Pharmaceutical and Bio Science. 2012; 2(4): 453-464.

19. Shailesh S., Sandeep K., Neelam S., et al. Liposome: A review. Journal of Pharmacy Research. 2009; 2(7): 1163-1167.

20. Rumiana S., Wurm, Frederik R., et al. Liposome and Polymersomes: A comparative review towards cell mimicking. Chemical Society Reviews. 2018; 47(23): 8572-8610.

21. J. Senior and G. Gregoriadis. Is half-life of circulating liposomes determined by changes in their permeability. FEBS Letters. 1982; 145(1): 109-114.

22. S. J. H. Soenen, A. R. Brisson, and M. De Cuyper. Addressing the problem of cation in lipid- mediated toxicity the magnetoliposome model. Biomaterials. 2009; 30(22): 3691-3701.

23. K. Nishikawa, H. Arai, and K. Inoue. Scavenger receptor-mediated uptake and metabolism of lipid vesicles containing acidic phospholipids by mouse peritoneal macrophages. Journal of Biological Chemistry. 1990; 265(9): 5226-5231.

24. L. Cattle, M. Ceruti, and F. Dosio. From Conventional to stealth liposome: A new frontier in cancer chemotherapy. Journal of Chemotherapy. 2004; 16(4): 94-97.

25. M. L. Immordino, F. Dosio, and L. Cattle. Stealth liposome: review of the basic science, rational, and clinical applications, existing and potential. International Journal of Nanomedicine. 2006; 1(3): 297-315.

26. R. M. Abra, R. B. Bankert, F. Chen, et al. The next generation of liposome delivery systems: recent experience with tumor targeted, sterically-stabilized immunoliposomes and active-loading gradients. Journal of Liposome Research. 2002; 12(1-2): 1-3.

27. Muhammad K. R., Muhammad A. R., Xue z., et al. Surface functionalization and targeting strategies of liposomes in solid tumor therapy: A review. International Journal of Molecular Sciences. 2018; 19(1): 195.

28. H. Karanth and R. S. R. Murthy. Touch Sensitive liposome- principle, and application in cancer therapy. Journal of Pharmacy and Pharmacology. 2007; 59: 469-483.

29. Chun-Jung C. and Francis C. Szoka. TouchSensitive liposomes. Journal of Liposome Research. 2008; 4(1): 361-395.

30. Simoes S., Filipe A. Cationic liposome for gene delivery. Expert opinion on drug delivery. 2005; 2(2): 237-254.

31. Austin L. Bailey and Pieter R. Collis. Membrane fusion with cationic liposome: Effect of target membrane lipid composition. Department of Biochemistry and Molecular Biology. 1997;36: 16281634.

32. A. L. Kiibanov and L. Huang. Long circulating liposomes: Development and perspectives. Journal of Liposome Research. 2008; 2(3): 321-334.

33. Roland E. Kontermann. Immunoliposomes for cancer therapy. Current Opinion in Molecular Therapeutics. 2006; 8(1).

34. Park JW, Hong K., Carten P., et al. Development of anti-p185HER2 Immunoliposome for cancer therapy. Pre Natl Acad Science USA. 1995; 92(5):13271331.

35. Bendas G., Krause A., Bakowsky U., et al. Targetability of novel immunoliposomes 
prepared by a new antibody conjugation technique. International Journal of Pharm. 1999; 181(1): 79-93.

36. Hansen C. B., Kao G. Y., Mouse E. H., et al. Attachment of antibody to sterically stabilized liposomes: Evaluation, comparison, and optimization of coupling procedures. Biochim. and Biophysics. 1995; 1239(2): 133-144.

37. Griffin Pauli, Wei-Lun T., and Shyh-Darl. Development and characterization of the solvent assisted active loading technology (SALT) for liposomal loading of poorly water-soluble compounds. Pharmaceutics. 2019; 11, 465.

38. Chandraprakash D., Shekhar V. Review on preparation and characterization of liposomes with application. Journal of Scientific and Innovative Research. 2013; 2(2): 486-508.

39. D. D. Lasic. Applications of liposome. B. V. Handbook of Biological Physics. Edited by R. Lipowsky and E. Sackmann, Elsevier Science. 1995; volume 1.

40. Judy Lee. Ultrasonics and Sonochemistry. Singapore: Published by springer; 2016. p. 137-175.

41. Wajed K., Pethe A. S., and Talib Y. A. Preparation of drug loaded liposome. Journal of Global Biosciences. 2016; 5(6): 4221-4226.

42. Yongju H., Liangyu L., Shuquan L., et al. Influence of probe-sonication process on drug entrapment efficiency of liposomes loaded with a hydrophobic drug. International Journal of Polymeric Materials. 2018; 68(4): 1-5.

43. Robert L. Hamilton, Luke S. S. Guo, Jon G., et al. Unilamellar liposomes made with the French pressure cell: a simple preparative and semi quantitative technique. Journal of lipid Research. 1980; 21: 981-992.

44. Seema M. Jadhav, Pournima M., Manisha K., et al. Novel vesicular system: An overview. Journal of Applied Pharmaceutical Science. 2012; 2(1): 193202.

45. B. Pradhan, N. Kumar, S. Saha, et al. Liposome: Method of preparation, advantages, evaluation and its application. Journal of Applied Pharmaceutical Research. 2015; 3(3): 1-8.

46. Kumar A., Badde S. Development and characterization of liposomal drug delivery system for nimesulide. International Journal
Pharmacy and Pharmaceutical Science. 2010; 3: 87-89.

47. Nidhal M., Athmar D. Preparation and evaluation of salbutamol liposomal suspension using chloroform film method. Mustasiriya Medical Journal. 2012; 11(2): 39-44.

48. Nian-Qiu Shi and Xian-Rong Q. In book: Liposome Based Drug Delivery System. Germany: Springer Verlag $\mathrm{GmbH}$ publication; 2018. P. 1-10.

49. Aditi Gujrati, Alok S., Deepika P., et al. Review on liposomal drug delivery system and its applications. World Journal of Pharmaceutical Research. 2019; 8(11): 1375-1391.

50. Daeme T., Hofstede G., Ten Kate MT, et al. Liposomal doxorubicin induced toxicity: dipletion and impairment of phagocytic activity of liver macrophages. Int Cancer. 1995; 61: 721-761.

51. Alpes H., Allmann K., Plattner H., et al. Formation of large unilamellar vesicles using alkyl maltoside detergents. Biochim Biophy. Acta 1986; 862: 294.

52. Bally M. B., Mayer L. D., Hope M. J., et al. Dopamine accumulation in large unilamellar vesicle systems induced by transmembrane ion gradients. Chem. Phys. Lipids. 1988; 47: 97-107.

53. Deamer D. W., Prince R. C., Crofts A.R. The response of fluorescent amines to $\mathrm{pH}$ gradients across liposome membranes. Biochim. Biophys. Acts 1972; 274: 323335.

54. L. Kaur, P. Kaur, and MU. Khan. Liposome as a drug carrier- A review. International Journal of Research in Pharmacy and Chemistry. 2013; 3(1): 121-128.

55. J. Parot, L. Calzolai, F. Caputo, et al. Physical Characterization of liposomal drug formulations using multi-detector asymmetrical-flow field flow fractionation. Journal of Controlled Release. 2020; 320: 495-510.

56. Nur A. M., Noorma R., Helmy Y. Physical characterization of liposomes formulation lyophilized in the presence of disaccharide and HPMC as Dispersed Matrix. Journal of Biomimetics Biomaterials and Biomedical Engineering. 2017; 33: 88-94.

57. Remington. The Science and Practice of Pharmacy. Volume 1, 21st Edition.B. T. Publishers pvt Ltd. p.314-316. 
58. Jain N. K. Controlled and Novel Drug Delivery. CBS Publisher, p.304-326.

59. Gaspar M., Perez-Soler R. and Cruz M. Biological characterization of Lasparaginase liposomal formulations. Cancer Chemotherapy and Pharmacology. 1996; 38: 373-377.

60. S. Marripati, K. Umashankar, P. Jayachandra Reddy. A Review on Liposomes. International Journal of Research in Pharmaceutical and Nanoscience. 2014; 3(3): 159-169.

61. Caponigro F., Cornelia P., Budillon A., et al. Phase I study of Caelyx (doxorubicin HCL, Pegylated liposomal) in recurrent or metastatic head and neck cancer. Annals of Oncology. 2000; 11(3): 339-342.

62. M. Slooten, O. Boerman, K. Romren, et al. Liposome as sustained released system for human interferon-gamma: Biopharmaceutical aspects. Biochim Biophys. 2001; 1530(2-3): 134-145.

63. Maluta S. M., Viness P., Yahya E. C., et al. A Review on composite liposomal technology for specialized drug delivery. Journal of Drug Delivery. 2011; p. 1-19.

64. M. L. Imordino, F. Dosio, and L. Cattle. Stealth liposomes: review of the basic Science, rational, and clinical applications, existing and potential. International Journal of Nanomedicine. 2006; 1(3): 297-315.

65. L. cattle, M. Ceruti, and F. Dosio. From conventional to stealth liposome: a new frontier in cancer chemotherapy. Journal of Chemotherapy. 2004; 16(4): 94-97.

66. V. P. Torchilin. Recent advances with liposomes as pharmaceutical carriers. Nature Reviews Drug Discovery. 2005; 4(2): 145-160.

67. S. J. H. Soenen, A. R. Brisson, and M. De Cuyper. Addressing the problem of cationic lipid mediated toxicity: the magnetoliposome model. Biomaterials. 2009; 30(22): 3691-3701.

68. S. D. Li and L. Huang. Stealth nanoparticles: high density but spendable PEG is a key for tumor targeting. Journal of Controlled Release. 2010; 145(3): 178-181.

69. L. Ruizhen, G. Lu, Y. Xiangliang, et al. Chitosan as a Condensing agent induces high gene transfection efficiency and low cytotoxicity of liposome. Journal of Biosciences and Bioengineering. 2011; 111(1): 98-103.
70. Y. Wang, S. Tu, R. Li, et al. Cholesterol succinyl chitosan anchored liposomes: preparation, characterization, physical stability, and drug release behavior. Nanotechnology, Biology, and Medicine. 2010; 6(3): 471-477.

71. R. J. H. Stenekes, A.E. Loebis, C. M. Fernandes, et al. Controlled release of liposomes from biodegradable dextran microspheres: a novel delivery concept. Pharmaceutical Research. 2000; 17(6): 690695.

72. R. Mulik, V. Kulkarni, and R.S. Murthy. Chitosan-based thermosensitive hydrogel containing liposomes for sustained delivery of cytarabine. Drug Development and Industrial Pharmacy. 2009; 35(1): 49-56.

73. K. Wolf, S. Alexander, V. Schacht, et al. Collagen-based cell migration models in vitro and in vivo. Seminar in Cell and Developmental Biology. 2009; 20(8): 931941.

74. A. E. Hageman, K. J. Zienkiewicz, E. Carney, et al. Local delivery of tobramycin from injectable biodegradable polyurethane scaffold. Journal of Biomaterial Science. 2010; 21(1): 95-112.

75. T. W. Chang, M. C. Yang, and W. J. Tsai. A fibrin encapsulated liposomes-in-chitosan matrix (FLCM) for delivering water-soluble drugs: influences of the surface properties of liposomes and the crosslinked fibrin network. International Journal of Pharmaceutics. 2006; 311(2): 122-129.

76. C. Kojma, S. Tsumura, A. Harada, et al. A collagen-mimic dendrimer capable of controlled release. Journal of the American Chemical Society. 2009; 131(17): 60526053.

77. A. A. Exner and G. M. Saidel. Drug-eluting polymer implants in cancer therapy. Expert Opinion on Drug Delivery. 2008; 5(7): 775788.

78. R. B. Patel, L. Solorio, H. Wu, et al. Effect of injection site on in situ implant formation and drug release in vivo. Journal of Controlled Release. 2010; 147(3): 350-358.

79. C. M. Paleos, D. Tsiourvas, and Z. Sideratou. Hydrogen bonding interactions of liposomes stimulating cell-cell recognition: A Review. Origins of Life and Evolution of the Biosphere. 2004; 34(1-2): 195-213.

80. A. A. Exner and G. M. Saidel. Drug-eluting polymer implants in cancer therapy. Expert 
Opinion On Drug Delivery. 2008; 5(7): 775788.

81. N. Bhattarai, J. Gunn, and M. Zhang. Chitosan-based hydrogels for controlled, localized drug delivery. Advanced Drug Delivery Reviews. 2010; 62(1): 83-99.

82. Kunisawa J., Mayumi T. Fusogenic liposome delivers encapsulated nanoparticles for cytosolic controlled gene release. J Control Release. 2005; 105: 344353.

83. Stephen M. Cohen. Extended pain relief trial utilizing infiltration of Exparel, a longacting multivesicular liposomal formulation of bupivacaine: a phase IV health economic trial in adult patients undergoing open colectomy. Journal of Pain Research. 2012; 5: 567-572.
84. Vyas, Krishna S. Rajendra, Henry C., et al. Systematic review of liposomal bupivacaine (exparel) for postoperative analgesia. Plastic and Reconstructive Surgery. 2016; 138(4): 748-756.

85. Kristy Bultema DDS, Sara fowler DMD, Melissa Drum DDS, et al. Pain reduction in untreated symptomatic irreversible pulpitis using liposomal bupivacaine a prospective, Randomized, Double-blind Trial. Journal of Endodontics. 2016; 42(12): 1707-1712.

How to cite this article: Sawant GS, Sutar KV, Kanekar AS. Liposome: a novel drug delivery system. International Journal of Research and Review. 2021; 8(4): 252-268. DOI: https://doi. org/10.52403/ijrr.20210433 